\title{
Clinical, Metabolic and Hormonal Profiles of Bangladeshi Adolescents with Polycystic Ovary Syndrome
}

\author{
ABM Kamrul-Hasan, ${ }^{1}$ Fatema Tuz Zahura Aalpona ${ }^{2}$ and Shahjada Selim ${ }^{3}$ \\ 1. Department of Endocrinology, Mymensingh Medical College, Mymensingh, Bangladesh; 2. Outpatient Department, Gynaecology \& \\ Obstetrics, Mymensingh Medical College Hospital, Mymensingh, Bangladesh; 3. Department of Endocrinology, Bangabandhu Sheikh Mujib \\ Medical University, Dhaka, Bangladesh.
}

DOI: https://doi.org/10.17925/EE.2021.17.1.54

$\mathrm{B}$ ackground: The features of polycystic ovary syndrome (PCOS) vary greatly among adolescent girls and adult women. Some of the features of PCOS may overlap with features of normal pubertal development in girls. Methods: This cross-sectional study was conducted among adolescents newly diagnosed with PCOS attending a tertiary hospital in Bangladesh. The relevant clinical, metabolic and hormonal profiles of 175 participants were evaluated. Results: The mean age of the study participants was $16.8( \pm 1.7)$ years. Oligomenorrhea was the predominant menstrual irregularity (88\%). More than one-quarter of participants (27.4\%) had a first-degree relative with PCOS, and $12 \%$ had a first-degree relative with type 2 diabetes. More than three-quarters (77.7\%) had acanthosis nigricans. The majority (69.1\%) were overweight (29.7\%) or obese (39.4\%), whereas 6.3\% were underweight. A total of $65.7 \%$ had abdominal obesity. One-fifth (20\%) of participants had pre-hypertension, and $3.4 \%$ were hypertensive. Around one-quarter (24\%) had abnormal glucose tolerance (prediabetes $21.1 \%$, diabetes $2.9 \%$ ) and the majority (90.9\%) had dyslipidaemia. The median Ferriman-Gallwey score was $12,94.9 \%$ of participants had hirsutism and $33.7 \%$ had biochemical hyperandrogenism. Metabolic syndrome was present in $42.3 \%$ of participants. Higher body mass index and presence of hirsutism were associated with higher risks of metabolic syndrome. Conclusions: The clinical, metabolic and hormonal profiles of Bangladeshi adolescents with PCOS highlight risk factors and the need for clinical vigilance with respect to metabolic disease.

\section{Keywords}

Polycystic ovary syndrome, adolescents, metabolic syndrome, hypertension, diabetes

Disclosure: ABM Kamrul-Hasan, Fatema Tuz Zahura Aalpona and Shahjada Selim have no financial or non-financial relationships or activities to declare in relation to this article.

Review Process: Double-blind peer review.

Compliance with Ethics: All procedures were followed in accordance with the responsible committee on human experimentation and with the Helsinki Declaration of 1975 and subsequent revisions, and informed consent was received from the patients involved in this study. Authorship: The named authors meet the International Committee of Medical Journal Editors (ICMJE) criteria for authorship of this manuscript, take responsibility for the integrity of the work as a whole, and have given final approval for the version to be published.

Access: This article is freely accessible at

touchENDOCRINOLOGY.com. (C) Touch Medical Media 2021

Received: 22 April 2020

Accepted: 24 June 2020

Published Online: 6 April 2021

Citation: touchREVIEWS in Endocrinology. 2021;17(1):54-8

Corresponding Author: ABM Kamrul-Hasan,

Department of Endocrinology, Mymensingh Medical

College, Mymensingh, 2200, Bangladesh.

E: rangassmc@gmail.com

Support: No funding was received for the publication of this manuscript.
Polycystic ovary syndrome (PCOS) is a heterogeneous androgen-excess disorder that presents with different degrees of reproductive and metabolic dysfunctions; it is also associated with insulin resistance and metabolic syndrome. ${ }^{1}$ The global prevalence estimates of PCOS in women of reproductive age are highly variable, ranging from $5 \%$ to $18 \% .{ }^{2}$ This could be due to the lack of a universal definition of PCOS, as the prevalence rates depend, to a great extent, on the criteria used to define this disorder.

Though PCOS can affect women at any stage of their reproductive age, the manifestations of PCOS may develop in adolescence but may not be diagnosed until well into adulthood. ${ }^{3}$ There is a lack of well-established diagnostic criteria for adolescent PCOS, as all PCOS diagnostic criteria are designed for adults. Diagnostic criteria include varying combinations of three features: oligo-/anovulation, clinical and/or biochemical hyperandrogenism and polycystic ovarian morphology. ${ }^{4-6}$ Normal adolescent physiology, including irregular cycles and acne, may mimic symptoms of PCOS. Besides, multi-follicular ovaries are a feature of normal puberty that subsides with the onset of a regular menstrual cycle and may be difficult to distinguish from PCOS morphology.? Therefore, the diagnosis of PCOS in adolescent girls is challenging, and the exact prevalence of PCOS in adolescent girls is mostly unknown. The prevalence of PCOS in Indian adolescents, using the Rotterdam criteria, has been reported as $9.13 \%{ }^{;}$however, the Rotterdam criteria has been found to be inappropriate for PCOS diagnosis in adolescents. In a recent meta-analysis, the prevalence of PCOS in adolescents based on the Rotterdam criteria was 11.04\% (95\% confidence interval [CI]: 6.84-16.09\%); based on the National Institute of Health criteria, it was 3.39\% (95\% Cl: 0.28-9.54\%); and based on Androgen Excess and Polycystic Ovary Syndrome Society, it was $8.03 \%$ (95\% Cl: 6.24-10.01\%). ${ }^{8}$ Recently, the Endocrine Society has suggested that the diagnosis of PCOS in adolescent girls should be made based on the presence of clinical and/or biochemical evidence of hyperandrogenism (after exclusion of other pathologies) in the presence of persistent menstrual irregularities. ${ }^{?}$

Identifying and treating adolescents with PCOS is of prime importance, as adult women with PCOS have a 10-fold increased risk of developing type 2 diabetes, and a 2-fold increased risk of metabolic syndrome. ${ }^{3}$ Additionally, PCOS is associated with worse reproductive outcomes, including subfertility and psychiatric comorbidities. ${ }^{1}$ Another reason that correct diagnosis is vital is 
that behavioural modification and lifestyle changes during adolescence play an essential role in the prevention of future complications and morbidity..$^{10}$ Data relating to the reproductive, metabolic and endocrine features of PCOS in adolescents in Bangladesh is lacking. The purpose of this study was to fill this knowledge gap.

\section{Materials and methods}

The protocol of the study obtained approval from the institutional review board. Informed consent was obtained from all patients in this study. This cross-sectional study was conducted among newly-diagnosed, consecutive adolescent patients (age 10-19 years) with PCOS attending the endocrinology outpatient department of Mymensingh Medical College Hospital, Mymensingh, Bangladesh, from January 2017 to December 2019. Those receiving drug treatmentfor diabetes, hypertension, dyslipidaemia and obesity, and those with pelvic inflammatory disease, any chronic debilitating illness or any malignancy, were excluded.

The diagnosis of PCOS in adolescent girls was made based on the presence of clinical and/or biochemical evidence of hyperandrogenism (after exclusion of other pathologies) in the presence of persistent menstrual irregularities. ${ }^{9}$ Oligomenorrhea was defined as menstrual cycle $>35$ days, or less than eight cycles per year after 2 years of menarche; primary amenorrhea as amenorrhea by age 15 years, or $>3$ years post thelarche; secondary amenorrhea as the cessation of menstruation for at least 6 months in a previously menstruating girl; and polymenorrhea as menstrual cycle $<21$ days.

A semi-structured, questionnaire-based interview on a one-to-one basis was conducted to collect detailed information on clinical presentation and family history. Height (to the nearest $0.1 \mathrm{~cm}$ ) was measured in all the individuals using wall-mounted stadiometers, and body weight (to the nearest $0.1 \mathrm{~kg}$ ) measured using electronic calibrated scales. Body mass index (BMI) was calculated from height and weight using the formula: height/weight ${ }^{2}$. Obesity status was determined by BMl categories applicable to Asian Indians. ${ }^{11}$ Waist circumference was measured (to the nearest $0.5 \mathrm{~cm}$ ) at the end of a gentle expiration midway between the lower rib margins and the iliac crests. Blood pressure (BP) was measured twice in each study participant by the auscultatory method using standard validated aneroid sphygmomanometer after at least 5 minutes of rest; two separate readings were taken at an interval of a minimum of 3 minutes, and the average of the two readings was used.

Hypertension and pre-hypertension were defined according to the Joint National Committee VII criteria. ${ }^{12}$ Hirsutism was assessed by the modified Ferriman-Gallwey (FG) score; a score of $\geq 8$ was the cutoff pointfor diagnosis of hirsutism. ${ }^{13}$ Oral glucose tolerance test (OGTT) with a 75-g glucose load was carried out in all participants after overnight fasting for at least 8 hours; fasting plasma glucose (FPG) and plasma glucose 2 hours after OGTT (PG 2H-OGTT) were measured by the glucose oxidase method using a fully automatic biochemistry analyser. Prediabetes and diabetes mellitus were diagnosed according to criteria described by the American Diabetes Association. ${ }^{14}$

Lipid profile was measured in all participants in fasting states using a fully automatic biochemistry analyser. Dyslipidaemia was defined according to cutoff points described in the Adult Treatment Panel III guideline..$^{15}$ Metabolic syndrome was diagnosed using the International Diabetes Federation consensus definition of metabolic syndrome in children and adolescents; abdominal obesity plus two or more of:

- raised blood pressure: systolic $\mathrm{BP} \geq 130 \mathrm{mmHg}$ or diastolic $\mathrm{BP} \geq 85$ $\mathrm{mmHg}$, or treatment of previously diagnosed hypertension;
- impaired fasting glucose: $F P G \geq 5.6 \mathrm{mmol} / \mathrm{L}$, or previously diagnosed type 2 diabetes;

- raised triglycerides ( $\geq 150 \mathrm{mg} / \mathrm{dL}$ ); or

- reduced high-density lipoprotein (HDL)-cholesterol (<50 mg/dL), or specific treatment for these lipid abnormalities.

Waist circumference $\geq 80 \mathrm{~cm}$ was defined as abdominal obesity for adolescents aged 17-19 years. ${ }^{16}$ In the adolescents (aged 10-16 years), waist circumference 290 th percentile (or adult cutoff if lower) for Indians was used to define abdominal obesity. ${ }^{10,17}$ Transabdominal pelvic ultrasonography (USG) was performed for all participants. Serum thyroid-stimulating hormone (TSH), total testosterone and prolactin were measured by automated hormone analyser using radioimmunoassay. Participants with abnormal TSH $(<0.3 \mu \mathrm{IU} / \mathrm{mL}$ or $>5.0 \mu \mathrm{lU} / \mathrm{mL}$ ) and serum prolactin $\geq 50 \mathrm{ng} / \mathrm{mL}$ were further excluded.

\section{Statistical analysis}

Statistical analyses were conducted using Statistical Packages for Social Sciences for Windows, version 23.0 software (SPSS Inc; Chicago, IL, USA). Categorical variables were presented as number (\%), measurable variables with normal distribution were presented as mean \pm standard deviation (SD), and those not following normal distribution were presented as median (interquartile range [IQR]). Logistic regression analysis was perfomed to identify the risk factors of metabolic syndrome in the study subjects. A p-value of $\leq 0.05$ was considered to be statistically significant.

\section{Results}

A total of 175 cases were included in the final analysis. The demographic and clinical parameters of the participants in this study are given in Table 1. Their mean age was 16.8 ( \pm 1.7 ) years. Oligomenorrhea (88.0\%) was the predominant type of menstrual irregularity, followed by secondary amenorrhea (6.9\%), polymenorrhea (2.9\%) and primary amenorrhea (2.3\%). Median FG score was 12 , and $94.9 \%$ of the participants had hirsutism. More than half of the participants $(59.4 \%$ ) gained weight in the 6 months preceding diagnosis of PCOS. More than one-quarter (27.4\%) had a first-degree relative with PCOS, and $12.0 \%$ had a first-degree relative with type 2 diabetes. More than three-quarters (77.7\%) had acanthosis nigricans. Only $24.6 \%$ had normal BMI, whereas $6.3 \%$ were underweight, $29.7 \%$ were overweight and $39.4 \%$ of the patients were obese.

The mean waist circumference of patients was $85.6( \pm 12.4) \mathrm{cm}$, and $65.7 \%$ had abdominal obesity. One-fifth (20.0\%) of participants had pre-hypertension and $3.4 \%$ were hypertensive. Around one-quarter (24.0\%) had abnormal glucose tolerance (prediabetes $21.1 \%$, diabetes 2.9\%). The majority of study participants (90.9\%) had dyslipidaemia. Metabolic syndrome was present in $42.3 \%$ of the patients (Table 2).

Hormonal and radiological parameters of the study participants are given in Table 3. Median total testosterone was $0.91 \mathrm{ng} / \mathrm{mL}$, and $33.7 \%$ of participants had biochemical hyperandrogenism (elevated total testosterone). Around one-quarter (24\%) had normal ovarian morphology in USG, whereas $45.1 \%$ had increased ovarian volume and $30.9 \%$ had a typical polycystic appearance on USG.

In logistic regression analysis, $\mathrm{BMI}$ and presence of hirsutism were found to increase the risk of metabolic syndrome in the study subjects (Table 4).

\section{Discussion}

PCOS in adolescents is an emerging problem. To our knowledge, this is the first study in Bangladesh, describing the clinical, metabolic and hormonal profiles of the adolescents with PCOS. In this study, most 
Table 1: Demographic and clinical characteristics of study participants

\begin{tabular}{|c|c|}
\hline Variables & $\mathrm{N}=175$ \\
\hline Age, mean \pm SD & $16.8 \pm 1.7$ \\
\hline \multicolumn{2}{|l|}{ Type of menstrual irregularity } \\
\hline Oligomenorrhea & $154(88.0)$ \\
\hline Primary amenorrhea & $4(2.3)$ \\
\hline Secondary amenorrhea & $12(6.9)$ \\
\hline Polymenorrhea & $5(2.9)$ \\
\hline Modified FG score, median (IQR) & $12(10-17)$ \\
\hline \multicolumn{2}{|l|}{ Hirsutism } \\
\hline Present & $166(94.9)$ \\
\hline Absent & $9(5.1)$ \\
\hline \multicolumn{2}{|c|}{ Gained weight in the 6 months preceding diagnosis } \\
\hline Yes & $104(59.4)$ \\
\hline No & $71(40.6)$ \\
\hline \multicolumn{2}{|c|}{ First-degree family member with PCOS } \\
\hline Present & $48(27.4)$ \\
\hline Absent & $127(72.6)$ \\
\hline \multicolumn{2}{|c|}{ First-degree family member with T2DM } \\
\hline Present & $21(12.0)$ \\
\hline Absent & $154(88.0)$ \\
\hline \multicolumn{2}{|l|}{ Acanthosis nigricans } \\
\hline Present & $136(77.7)$ \\
\hline Absent & $39(22.3)$ \\
\hline $\mathrm{BMI}$, mean kg/m² $\pm \mathrm{SD}$ & $26.3 \pm 5.6$ \\
\hline \multicolumn{2}{|l|}{ BMI category } \\
\hline Underweight & $11(6.3)$ \\
\hline Normal weight & $43(24.6)$ \\
\hline Overweight & $52(29.7)$ \\
\hline Obese & $69(39.4)$ \\
\hline
\end{tabular}

Data are presented as $n(\%)$ unless stated otherwise.

$B M I=$ body mass index; $F G$ = Ferriman-Gallwey; $I Q R$ = interquartile range;

PCOS = polycystic ovary syndrome; $S D=$ standard deviation; T2DM = type 2 diabetes mellitus.

(94.9\%) of the patients had hirsutism. More than two-thirds (69.1\%) were either overweight or obese and almost a similar number (65.7\%) had abdominal obesity. A very high frequency of dyslipidaemia (90.9\%) was observed and the prevalence of metabolic syndrome was also considerably high (42.3\%).

In adolescents, natural maturity characteristics usually overlap with signs and symptoms of PCOS. This issue leads to particular diagnostic problems, and based on the evidence, the debate on the aetiopathogenesis, diagnostic criteria and suggestions for PCOS in adolescents continues. ${ }^{8}$ The diagnostic challenge in adolescents may be due to many reasons, such as the higher rate of physiologic anovulatory cycles, irregular menses during the first 2 years following menarche, and the presence of acne in this age group..$^{18}$ Due to these transitory symptoms and signs mimicking PCOS during adolescence, care must be taken to avoid premature labelling of a case as PCOS to avoid overtreatment and psychological stress. The presence of oligomenorrhea among adolescent girls 2 years post-menarche is a good screening indicator to diagnose a probable case of PCOS. Diagnosis is confirmed if there is clinical and/ or biochemical evidence of hyperandrogenism in the presence of persistent menstrual irregularities. ${ }^{9}$ Worldwide, PCOS in adolescents is
Table 2: Metabolic characteristics of study participants

\begin{tabular}{|c|c|}
\hline Variables & $\mathrm{N}=175$ \\
\hline Waist circumference, mean $\mathrm{cm} \pm \mathrm{SD}$ & $85.6 \pm 12.4$ \\
\hline \multicolumn{2}{|l|}{ Abdominal obesity, $n$ (\%) } \\
\hline Present & $115(65.7)$ \\
\hline Absent & $60(34.3)$ \\
\hline Systolic BP, mean $\mathrm{mmHg} \pm \mathrm{SD}$ & $114 \pm 11$ \\
\hline Diastolic BP, mean $\mathrm{mmHg} \pm \mathrm{SD}$ & $74 \pm 8$ \\
\hline \multicolumn{2}{|l|}{ BP status, n (\%) } \\
\hline Normal & $134(76.6)$ \\
\hline Pre-HTN & $35(20.0)$ \\
\hline HTN & $6(3.4)$ \\
\hline FPG, mean $\mathrm{mmol} / \mathrm{L} \pm \mathrm{SD}$ & $4.9 \pm 0.8$ \\
\hline PG $2 \mathrm{H}-\mathrm{OGTT}$, mean $\mathrm{mmol} / \mathrm{L} \pm \mathrm{SD}$ & $6.5 \pm 1.6$ \\
\hline \multicolumn{2}{|l|}{ Glycaemic status, n (\%) } \\
\hline Normoglyacemia & $133(76.0)$ \\
\hline Prediabetes & $37(21.1)$ \\
\hline Diabetes mellitus, $n$ (\%) & $5(2.9)$ \\
\hline Serum triglyceride, mean $\mathrm{mg} / \mathrm{dL} \pm \mathrm{SD}$ & $136.5 \pm 47.6$ \\
\hline Serum total cholesterol, mean $\mathrm{mg} / \mathrm{dL} \pm$ SD & $164.9 \pm 31.4$ \\
\hline Serum LDL cholesterol, mean $\mathrm{mg} / \mathrm{dL} \pm \mathrm{SD}$ & $99.4 \pm 25.6$ \\
\hline Serum HDL cholesterol, mean $\mathrm{mg} / \mathrm{dL} \pm \mathrm{SD}$ & $37.7 \pm 7.5$ \\
\hline \multicolumn{2}{|l|}{ Dyslipidaemia, n (\%) } \\
\hline Present & $159(90.9)$ \\
\hline Absent & $16(9.1)$ \\
\hline \multicolumn{2}{|l|}{ Metabolic syndrome, n (\%) } \\
\hline Present & $74(42.3)$ \\
\hline Absent & $101(57.7)$ \\
\hline
\end{tabular}

$B P=$ blood pressure; $F P G=$ fasting plasma g/ucose; $H D L=$ high-density lipoprotein; $H T N=$ hypertension; $L D L=$ low-density lipoprotein; $O G T T=$ oral glucose tolerance test, PG 2H-OGTT = plasma g/ucose 2-hour after OGTT; $S D$ = standard deviation.

Table 3: Endocrine and radiological characteristics of study participants

\begin{tabular}{|l|l|}
\hline Variables & $\mathrm{N}=175$ \\
\hline Serum testosterone, median $\mathbf{n g} / \mathbf{m L}(\mathbf{I Q R})$ & $0.91(0.51-1.60)$ \\
\hline Biochemical hyperandrogenism, $\mathbf{n}(\%)$ & \\
\hline Present & $59(33.7)$ \\
\hline Absent & $116(66.3)$ \\
\hline Serum prolactin, median $\mathbf{n g} / \mathbf{m L}$ (IQR) & $14.97(9.63-22.43)$ \\
\hline Serum TSH, median $\boldsymbol{\text { IIU/mL (IQR) }}$ & $1.42(0.91-2.36)$ \\
\hline Ovarian morphology in USG, $\mathbf{n}$ (\%) & \\
\hline Normal & $42(24.0)$ \\
\hline Typical PCO & $54(30.9)$ \\
\hline Increased ovarian volume & $79(45.1)$ \\
\hline
\end{tabular}

$I Q R$ = interquartile range; $P C O=$ polycystic ovary; $T S H$ = thyroid-stimulating hormone USG = ultrasonography.

an emerging problem that needs careful assessment, timely intervention and appropriate treatment. ${ }^{19}$

In this study, $88 \%$ of the patients had oligomenorrhea, $2.3 \%$ had primary amenorrhea, 6.9\% had secondary amenorrhea (6.9\%), and 2.9\% had polymenorrhea. In Indian adolescents diagnosed with PCOS, Balaji et al. found that $28 \%$ of girls have oligomenorrhea and $18 \%$ had secondary 
Table 4: Risk factors of metabolic syndrome in the study subjects

\begin{tabular}{|c|c|c|c|}
\hline Risk factor & $p$-value & Odds ratio & $95 \%$ confidence interval for odds ratio \\
\hline Age & 0.889 & 1.016 & $0.814-1.268$ \\
\hline BMl & $<0.001$ & 1.233 & $1.104-1.377$ \\
\hline First-degree family member with PCOS & 0.070 & 0.302 & $0.083-1.101$ \\
\hline First-degree family member with T2DM & 0.183 & 1.804 & $0.757-4.297$ \\
\hline Hirsutism & 0.015 & 11.337 & $1.611-79.768$ \\
\hline Biochemical hyperandrogenism & 0.964 & 0.981 & $0.414-2.321$ \\
\hline PCO or increased ovarian volume in USG & 0.764 & 0.873 & $0.361-2.111$ \\
\hline
\end{tabular}

Data presented as mean $\pm S D$ unless stated otherwise.

P-value by Student's t-test, Chi-square test or Mann-Whitney $U$ test as applicable.

$B M I=$ body mass index; $P C O=$ polycystic ovary; $P C O S=$ polycystic ovary syndrome; T2DM = type 2 diabetes mellitus; USG = ultrasonography.

amenorrhea. ${ }^{20}$ In the same country, Nidhi et al. reported the prevalence of oligo/amenorrhea to be $97.62 \%$ amongst adolescents with PCOS. ${ }^{3}$ Hirsutism was present in almost all (94.9\%) of the patients in our study. The prevalence of hirsutism observed in India among similar patients was variable and lower than our observations (21\% by singh et al., $52 \%$ by Balaji et al. and $72.26 \%$ by Desai et al.). ${ }^{10,20,21}$ The modified FG score is a visual grading system; as it is subjective in nature, it may have significant inter- and intra-observer variability. Another important determining factor of hirsutism is the race of the subject and it is proposed that the same modified FG of $\geq 8$ is not suitable for defining hirsutism in all races. ${ }^{22}$ All of these factors might influence the varying prevalence of hirsutism observed in the above-mentioned studies.

In this study, $27.4 \%$ of the girls had a first-degree family member with PCOS. Singh et al. found that $43 \%$ of patients with PCOS in India had a first-degree relative with PCOS. ${ }^{10}$ Kahsar-Miller et al. observed that $24 \%$ of mothers and $32 \%$ of sisters of patients with PCOS also had PCOS. ${ }^{23}$ Evidence has also shown that family history of type 2 diabetes is significantly higher among women with PCOS and is strongly associated with an increased risk of diabetes and metabolic disorders in women with PCOS. ${ }^{24}$ Even non-obese brothers and sisters (without PCOS) of patients with PCOS are found to have higher insulin resistance and related metabolic defects. ${ }^{25,26}$ In this study, $12 \%$ of the participants had a first-degree relative with type 2 diabetes. Desai et al. found that $37.81 \%$ of the adolescents with PCOS have a family history of diabetes mellitus. ${ }^{21}$ These findings indicate that there is a genetic predisposition for PCOS. Acanthosis nigricans, which is a clinical marker of insulin resistance, was observed in $77.7 \%$ of the patients in our study. This frequency is higher than that (56.50\%) observed in young Pakistani women with PCOS, as studied by Tabassum et al. ${ }^{27}$ This may be due to the racial variability in the prevalence of acanthosis nigricans; the prevalence of acanthosis nigricans is higher in black women compared with white women. ${ }^{28}$

In our study, the majority of the patients were overweight or obese $(29.7 \%$ and $39.4 \%$, respectively), though a small portion was underweight (6.3\%). In a study by Balaji et al. in Indian adolescents with PCOS, the prevalence of underweight, normal weight, overweight and obesity was 14\%, $71 \%$, $9 \%$ and $2 \%$, respectively. ${ }^{20}$ In another study by singh et al., $50 \%$ of such patients were not obese or overweight, $43 \%$ were overweight and $7 \%$ were obese. ${ }^{10}$ This suggests that obesity status in PCOS is variable, even in similar populations, and may be influenced by the cutoff values of BMl used to define overweight and obese people.

since adolescents with PCOS are, as with their adult counterparts, insulin resistant, they are at increased risk for metabolic syndrome.
This has been confirmed by several observational studies, which have reported that adolescents with PCOS are at increased risk for metabolic syndrome compared with controls, and metabolic syndrome prevalence in an adolescent PCOS cohort was found to be at least threefold greater than normal adolescents when adjusted for BMI. ${ }^{29-31}$ In the current study, metabolic syndrome was found in $42.3 \%$ of the study participants. The reported prevalence of metabolic syndrome in adolescents with PCOS greatly varies in different regions; the prevalence is reported to be $33.3 \%$ in Iran, 26\% in Turkey, and 37\% in the USA. ${ }^{30,32,33}$ The prevalence of metabolic syndrome in the South Asian population, including Bangladesh, is higher than in western countries and many other parts of the world; this is also true for the adolescent age group. ${ }^{34}$ There are no universal criteria for defining metabolic syndrome in adolescents, and the use of different criteria in different studies and countries is likely to be the reason for such variation in the aforementioned studies.

Researchers have found significantly higher frequencies of metabolic syndrome components, such as hypercholesterolaemia, hypertriglyceridaemia and elevated BP in adolescents with PCOS than in their healthy counterparts. ${ }^{18}$ Joshi et al., in their study, observed that $7.1 \%$ of Indian adolescents with PCOS had hypertension. ${ }^{19}$ Coviello et al., in the USA, found $26 \%$ of adolescent girls diagnosed as PCOS to have hypertension. ${ }^{33}$ Coviello et al. used paediatric diagnostic criteria for defining hypertension that may be associated with the higher prevalence of hypertension in their study. Abnormal glucose tolerance was observed in $24 \%$ (21.9\% prediabetes, $2.9 \%$ diabetes) of the participants of the current study. The prevalence of impaired fasting glucose, impaired glucose tolerance and type 2 diabetes were reported to be 3.0\%, 15.2\%, and $1.5 \%$, respectively, among adolescents with PCOS in the USA. ${ }^{35}$ On the other hand, the prevalence of impaired fasting glucose (8.91\%), impaired glucose tolerance (29.16\%) and diabetes (3.5\%) were higher in Indian adolescents with PCOS according to the findings of Ashraf et al..$^{36}$

$\mathrm{BMI}$ is the most important variable that precludes the presence of metabolic syndrome. ${ }^{18,37}$ In this study, higher BMI was found to significantly increase the risk of metabolic syndrome. Similarly to Bhattacharya et al., we observed no influence of age on the presence of metabolic syndrome. Mandrelle et al. identified age as an independent risk factor for metabolic syndrome in PCOS. ${ }^{37,38}$ In this study, presence of hirsutism was associated with increased risk of metabolic syndrome, whereas elevated total testosterone was not. The exact inter-relationship between androgenic and metabolic problems of PCOS remains unresolved. A large Chinese study reports that hyperandrogenaemia, not hirsutism, is independently associated with type 2 diabetes mellitus. ${ }^{39}$ Others also 
demonstrate hyperandrogenism-related metabolic syndrome. ${ }^{40}$ On the other hand, the level or degree of hyperandrogenism did not alter the metabolic parameters in the study conducted by Forrester-Dumont et al. ${ }^{41}$ Plasma testosterone was also not statistically linked to the occurrence of metabolic syndrome among Sri Lankans with PCOS. ${ }^{22}$ Among adolescent Australian girls with PCOS, neither menstrual disturbances nor polycystic ovary morphology were found to be related to insulin resistance or metabolic syndrome. ${ }^{43}$ Similarly, we observed no influence of polycystic ovary morphology in pelvic USG in the presence of metabolic syndrome.

This study has some limitations. It was a single-centre study; the sample may not represent the whole country. No healthy control group was included, meaning it was not possible to compare these results to patients without PCOS. Nevertheless, this is the first study in this country describing the clinical, metabolic and hormonal parameters in adolescent patients with PCOS and may serve as the basis for further large-scale, multi-centre studies in this subject.

\section{Conclusion}

Adolescents with PCOS share many of the same clinical and metabolic abnormalities as their adult counterparts. Assessment of comorbidities of PCOS in these adolescent girls with particular emphasis on the metabolic derangements is of great importance for timely therapeutic intervention to prevent future complications. $]$
1. Fauser $\mathrm{BC}$, Tarlatzis $\mathrm{BC}$, Rebar RW, et al. Consensus on women's health aspects of polycystic ovary syndrome (PCOS): the Amsterdam ESHRE/ASRM-Sponsored 3rd PCOS Consensus Workshop Group. Fertil Steril. 2012;97:28-38.

2. Ding T, Hardiman PJ, Petersen I, et al. The prevalence of polycystic ovary syndrome in reproductive-aged women of different ethnicity: a systematic review and meta-analysis. Oncotarget. 2017:8:96351-8.

3. Nidhi R, Padmalatha V, Nagarathna R, et al. Prevalence of polycystic ovarian syndrome in Indian adolescents. J Pediat Adolesc Gynecol. 2011:24:223-7.

4. Zawadzki JK, Dunaif A. Diagnostic criteria for polycystic ovary syndrome: towards a rational approach. In: Dunaif $A G$ Haseltine F (eds). Polycystic Ovary Syndrome, Boston MA: Blackwell Scientific, 1992;377-84

5. Rotterdam ESHRE/ASRM-Sponsored PCOS Consensus Workshop Group. Revised 2003 consensus on diagnostic criteria and long-term health risks related to polycystic ovary syndrome. Fertil Steril. 2004;81:19-25.

6. Azziz R, Carmina E, Dewailly D, et al. The Androgen Excess and PCOS Society criteria for the polycystic ovary syndrome: the complete task force report. Fertil Steril. 2009;91:456-88.

7. Bridges NA, Cooke A, Healy MJ, et al. Standards for ovarian volume in childhood and puberty. Fertil Steril. 1993;60:456460.

Naz MSG, Tehrani FR, Majd HA, et al. The prevalence of polycystic ovary syndrome in adolescents: A systematic review and meta-analysis. Int I Reprod Biomed (Yazd). 2019:17:533-42.

9. Legro RS, Arslanian SA, Ehrmann DA, et al.: Endocrine Society. Diagnosis and treatment of polycystic ovary syndrome: an Endocrine Society clinical practice guideline. $J$ Clin Endocrino Metab. 2013:98:4565-92.

10. Singh A, Vijaya K, Sai Laxmi K. Prevalence of polycystic ovarian syndrome among adolescent girls: a prospective study. Int J Reprod Contracept Obstet Gynecol. 2018;7:4375-8.

1. WHO Expert Consultation. Appropriate body-mass index for Asian populations and its implications for policy and intervention strategies. Lancet. 2004;363:157-63.

12. Chobanian AV, Bakris GL, Black HR, et al. The seventh report of the Joint National Committee on Prevention, Detection Evaluation and Treatment of High Blood Pressure: the JNC 7 report. JAMA. 2003:289:2560-72.

13. Hatch R, Rosenfield RL, Kim MH, et al. Hirsutism: implications, Hatch R, Rosenfield RL, KIm MH, et al. Hirsutism: implications,
etiology, and management. Am J Obstet Gynecol. 1981:140:815-30.

14. American Diabetes Association. 2. Classification and diagnosis of diabetes: standards of medical care in diabetes-2019. Diabetes Care. 2019;42:S13-28.

15. National Cholesterol Education Program (NCEP) Expert Panel on Detection, Evaluation, and Treatment of High Blood Cholesterol in Adults (Adult Treatment Panel III). Third report of the National Cholesterol Education Program (NCEP) Expert Panel on Detection, Evaluation, and Treatment of High Blood Cholesterol in Adults (Adult Treatment Panel III) final report. Circulation. 2002;106:3143-421.
16. International Diabetes Federation. IDF consensus definition of metabolic syndrome in children and adolescents. 2007. Available at: www.idf.org/e-library/consensus-statements/61idf-consensus-definition-of-metabolic-syndrome-in-childrenand-adolescents.html (accessed 15 July 2020).

17. Khadilkar A, Ekbote V, Chiplonkar $\mathrm{S}$, et al. Wais circumference percentiles in 2-18 year old Indian children. Pediatr. 2014;164:1358-62 e2.

18. Cirik DA, Dilbaz B. What do we know about metabolic syndrome in adolescents with PCOS? J Turk Ger Gynecol Assoc. 2014:15:49-55.

19. Joshi B, Mukherjee S, Patil A, et al. A cross-sectional study of polycystic ovarian syndrome among adolescent and young girls in Mumbai, India. Indian J Endocrinol Metab. 2014:18:317-24

20. Balaji S, Amadi C, Prasad S, et al. Urban rural comparisons of polycystic ovary syndrome burden among adolescent girls in a hospital setting in India. Biomed Res Int. 2015;2015:158951

21. Desai NA, Tiwari RY, Patel SS. Prevalence of polycystic ovary syndrome and its associated risk factors among adolescent and young girls in Ahmedabad region. Int J Pharm Pract. 2018;11:119-25.

22. Yildiz BO, Bolour S, Woods K, et al. Visually scoring hirsutism. Hum Reprod Update. 2010;15:51-64.

23. Kahsar-Miller MD, Nixon C, Boots LR, et al. Prevalence of polycystic ovary syndrome (PCOS) in first-degree relatives of patients with PCOS. Fertil Steril. 2001:75:53-8.

24. Ehrmann DA, Kasza K, Azziz R, et al. Effects of race and family history of type 2 diabetes on metabolic status of women with polycystic ovary syndrome. I Clin Endocrinol Metab. 2005:90:66-71.

25. Baillargeon JP, Carpentier AC. Brothers of women with polycystic ovary syndrome are characterised by impaired glucose tolerance, reduced insulin sensitivity and related metabolic defects. Diabetologia. 2007;50:2424-32.

26. Diamanti-Kandarakis E, Alexandraki K, Bergiele A, et a Presence of metabolic risk factors in non-obese PCOS sisters: evidence of heritability of insulin resistance. J Endocrinol Invest 2004;27:931-6.

27. Tabassum R, Imtiaz F, Sharafat $\mathrm{S}$, et al. Prevalence and clinical profile of insulin resistance in young women of poly cystic ovary syndrome: a study from Pakistan. Pak J Med Sci. 2013:29:593-6.

28. Stuart CA, Smith MM, Gilkison CR, et al. Acanthosis nigricans among native Americans: an indicator of high diabetes risk. Am J Public Health. 1994;84:1839-42.

29. Palmert MR, Gordon CM, Kartashov Al, et al. Screenin for abnormal glucose tolerance in adolescents with polycystic ovary syndrome. J Clin Endocrinol Metab. 2002;87:1017-23.

30. Rahmanpour H, Jamal L, Mousavinasab SN, et al. Association between polycystic ovarian syndrome, overweight, and metabolic syndrome in adolescents. $J$ Pediatr Adolesc Gynecol. 2012;25:208-12

31. Cook $S$, Weitzman $M$, Auinger $P$, et al. Prevalence of metabolic syndrome phenotype in adolescents: findings from the third National Health and Nutrition Examination Survey, 1988-1994. Arch Pediatr Adolesc Med. 2003;157:821-7.

32. Çalışkan $\mathrm{E}$, Killç T, Bodur $\mathrm{H}$, et al. The frequency of metabolic syndrome in women with polycystic ovaries at reproductive ages and comparison of different metabolic syndrome diagnostic criteria. J Turk Ger Gynecol Assoc. 2007:8:402-7.

33. Coviello AD, Legro RS, Dunaif A. Adolescent girls with polycystic ovary syndrome have an increased risk of the metabolic syndrome associated with increasing androgen levels independent of obesity and insulin resistance. J Clin Endocrino Metab. 2006;91:492-7.

34. Pandit K, Goswami S, Ghosh S, et al. Metabolic syndrome in South Asians. Indian J Endocrinol Metab. 2012;16:44-55

35. Flannery CA, Rackow B, Cong X, et al. Polycystic ovary syndrome in adolescence: impaired glucose tolerance occurs across the spectrum of BMI. Pediatr Diabetes. 2013;14:42-9.

36. Ashraf $\mathrm{G}$, Khurana M, Eunice $\mathrm{M}$, et al. Prevalence of glucose intolerance among adolescent and young women with polycystic ovary syndrome in India. Indian I Endocrinol Metab. 2004;6:9-14

37. Bhattacharya SM, Jha A. Prevalence and risk of metabolic syndrome in adolescent Indian girls with polycystic ovary syndrome using the 2009 'joint interim criteria'. J Obstet Gynaecol Res. 2011;37:1303-7.

38. Mandrelle K, Kamath MS, Bondu DJ, et al. Prevalence of metabolic syndrome in women with polycystic ovary syndrome attending an infertility clinic in a tertiary care hospital in south India. J Hum Reprod Sci. 2012;5:26-31.

39. Zhao X, Zhong J, Mo Y, et al. Association of biochemical hyperandrogenism with type 2 diabetes and obesity in chinese women with polycystic ovary syndrome. Int I Gynaecol obstet. 2010;108:148-51

40. Shroff R, Syrop CH, Davis W, et al. Risk of metabolic complications in the new PCOS phenotypes based on the Rotterdam criteria Fertil Steril. 2007:88:1389-95.

41. Forrester-Dumont K, Galescu O, Kolesnikov A, et al. Hyperandrogenism does not influence metabolic parameters in adolescent girls with PCOS. Int J Endocrinol. 2012:2012:434830.

42. Wijeyaratne $\mathrm{CN}$, Seneviratne R de A, Dahanayake $\mathrm{S}$, et al. Phenotype and metabolic profile of South Asian women with polycystic ovary syndrome (PCOS): results of a large database from a specialist Endocrine Clinic. Hum Reprod. 2011;26:202-13.

43. Hart R, Doherty DA, Mori T, et al. Extent of metabolic risk in adolescent girls with features of polycystic ovary syndrome Fertil Steril. 2011;95:2347-53, 2353.e1. 